\title{
Capitation Payment Method
}

National Cancer Institute

\section{Source}

National Cancer Institute. Capitation Payment Method. NCI Thesaurus. Code C70684.

The payment mechanism that is used to reimburse healthcare providers for the care provided to a patient in form of a regular periodic fee, which is paid to compensate some or all services rendered by all providers involved for all conditions affecting a particular patient. 